\author{
Alban L. Denys \\ Thierry De Baere \\ Viseth Kuoch \\ Benoit Dupas \\ Patrick Chevallier \\ David C. Madoff \\ Pierre Schnyder \\ Francesco Doenz
}

\section{Radio-frequency tissue ablation of the liver: in vivo and ex vivo experiments with four different systems}

Received: 8 January 2003

Revised: 7 April 2003

Accepted: 2 May 2003

Published online: 27 August 2003

(C) Springer-Verlag 2003

\author{
A. L. Denys $(\bowtie) \cdot$ P. Chevallier \\ P. Schnyder · F. Doenz \\ Department of Radiology \\ and Interventional Radiology, \\ Centre Hospitalier Universitaire Vaudois, \\ 1011 Lausanne, Switzerland \\ e-mail: Alban.Denys@chuv.hospvd.ch \\ Tel.: +41-21-3144470 \\ Fax: +41-21-3144443 \\ T. D. Baere · V. Kuoch \\ Department of Interventional Radiology, \\ Institut Gustave Roussy, \\ 39 Rue Camille Desmoulins, \\ 94805 Villejuif, France \\ B. Dupas \\ Department of Radiology, \\ CHU Hotel Dieu, \\ Place Alexis Ricordeau, \\ 44093 Nantes Cedex 1, France \\ D. C. Madoff \\ Department of Interventional Radiology, \\ University of Texas, \\ M.D. Anderson Cancer Center, \\ Houston, TX, 77030, USA
}

\begin{abstract}
The aim of this study was to test the efficacy of four different radio-frequency ablation (RFA) systems in normal hepatic parenchyma in large animals. The RFA was applied to pig livers in vivo and to calf livers ex vivo using the Radionics cluster needle, RITA starburst XL needle, Radiotherapeutics Le Veen 4.0 needle, and the Berchtold 14-G saline-perfused 15-mm active-tip needle based on constructor specifications. The volume of tissue coagulation from RF was calculated from measurements of the vertical diameter (Dv) and transverse diameter (Dt). Lesion shape was characterized using the ratio between Dt/Dv. Radiotherapeutics and RITA produced in vivo lesion volume of $42 \pm 10$, $39 \pm 4 \mathrm{~cm}^{3}$ with a reproducible spherical shape (Dt/Dv of $1.01 \pm 0.16$ and $0.97 \pm 0.1$, respectively). Radionics produced in vivo RF lesions volume of $29 \pm 11 \mathrm{~cm}^{3}$ with an ovoid shape (Dt/Dv 0.88 \pm 0.09 ). The RF lesions with the Berchtold device could not
\end{abstract}

be assessed in vivo as 5 of 8 animals died during treatment. Ex vivo RF lesions had similar volumes with each system; however, the Radiotherapeutics device produced more reproducible shaped lesions than the other systems. In our experimental study, we found no difference between expandable needle systems in vivo. Cooled needles produced slightly smaller and ovoid shape in vivo lesions.

Keywords Experimental study . Radio-frequency ablation - Liver . Electrode

\section{Introduction}

Image-guided tumor ablation has become increasingly popular in recent years. While various percutaneous ablative techniques currently exist for the treatment of selected patients with hepatic malignancy, RFA is currently the most widely used technique [1]. To perform RFA, a needle electrode is inserted into the tumor under imaging guidance. This probe emits alternative current with frequencies ranging from 450 to $500 \mathrm{KHz}$ that in- duce ionic agitation, thus producing heat. At temperatures above $60^{\circ} \mathrm{C}$, cells die instantly from protein coagulation and dehydration.

The first RF needle electrodes available produced small volumes of necrosis with destruction in the liver limited to the maximal diameter perpendicular to the needle axis less than $1.6 \mathrm{~cm}$ after a single focus of RF delivery [2]. For this reason, complete tumor destruction usually necessitated multiple, overlapping RF deliveries, limiting the use of this technique. To avoid this problem, 
needle electrodes have been optimized in different ways, i.e., expandable needles, cooled cluster needle, saline enhanced or wet electrode, and some combined forms such as cooled-wet or expandable-wet electrodes, in an attempt to increase the area of tissue destruction obtained with one RF delivery [3, 4, 5, 6, 7, 8, 9, 10, 11]. Furthermore, the control of RF delivery according to impedance, time, and/or temperature is different from one system to another.

The purpose of this study was to compare the efficacy in normal liver parenchyma in vivo and ex vivo of four commercially available RF delivery systems (needle and generator; time of study: from June to November 2001) from Bertchtold (Tuttlingen, Germany), Radionics (Burlington, Mass.), Radiotherapeutics (Sunnyvale, Calif.), and RITA (Mountain View, Calif.). The RF protocols of energy deposits were made according to manufacturers' specifications.

\section{Materials and methods}

\section{Study design}

Experiments were performed in the interventional radiology research center of the national center for agronomic research. Large White Swine weighing between 80 and $100 \mathrm{~kg}$ were used in accordance with legislation governing animal care. After pre-medication with Ketamine, 21 animals were intubated and anesthetized with methoxyflurane (1-2\%). Cardiac and respiratory parameters were monitored and maintained throughout the procedures. After careful skin preparation, grounding pads were placed on the thigh according to manufacturer's specifications. Median laparotomies with intra-operative ultrasound guidance (7.5- $\mathrm{MHz}$ probe) were performed in all animals to allow for precise positioning of the RF electrodes. The RF electrodes were placed by one of the two investigators (A.D. or T.D.B.), into the thicker portion of the liver and away from large vessels, as previously described [3]. Due to the relatively large size of the RF lesions produced when compared with the remainder of the liver, only one to three lesions could be created per animal liver. Radio-frequency units and the order in which each lesion was produced were randomly assigned.

The animals were killed and the livers harvested immediately after the experiments. Specimens were cut along the needle axis into 5-mm-thick slices. The RF areas of destruction were measured in two perpendicular directions, one along the needle axis called vertical diameter (Dv) and the other perpendicular to it called transverse diameter (Dt) These measurements were performed in consensus of two investigators (A.D., T.D.B.). Macroscopic changes in specimens have been demonstrated to correlate well with coagulation necrosis at histopathological examination [2].

The ex vivo study was conducted on explanted calf livers at room temperature. One grounding pad was positioned more than $20 \mathrm{~cm}$ away from the distal tip of the RF electrode. The tip of the needle electrode was at least $5 \mathrm{~cm}$ deep within the liver parenchyma (lesions were created for each system, 5-8 lesions were created in each calf liver). Lesion size measurements were performed in the same manner as in the in vivo experiment.

\section{Protocols of RF delivery}

Four RF systems were evaluated during this study which included Bertchtold Elektrotom (Tuttlingen, Germany), Radionics (Burling- ton, Mass.), Radiotherapeutics (Sunnyvale, Calif.), and RITA (Mountain View, Calif.; Fig. 1). Lesions were created according to specific protocols defined by each of the four RF manufacturers with regard to their own RF device. The systems were operated during the experiment by experts experienced in running the machines who were chosen by each company.

For this study, a Berchtold Elektrotom 60 W 106 HITT device was used in association with a $14-\mathrm{G}$ single needle electrode. The needle has an exposed tip $15 \mathrm{~mm}$ long in which there are three groups of two side holes each, placed at $120^{\circ}$ angle from each other. The needle was connected to an infusing pump that was set to deliver $100 \mathrm{ml} / \mathrm{h}$ of normal saline at room temperature through the needle side holes. Care was taken to begin the infusion immediately prior to the liver puncture in order to avoid obstruction of the needle electrode side holes during placement. According to company recommendations, three separate 5-min RF deliveries (total RF time: $15 \mathrm{~min}$ ) with a power of $50 \mathrm{~W}$ was performed at each needle position. Between each 5-min RF delivery, the RF was turned off for 2-min intervals.

The Radionics RF system used for this study was a 3CC1 Radionics generator that produces a maximum power of $200 \mathrm{~W}$ through a $17-\mathrm{G}$ triple cluster-cooled needle electrode. The triple cluster needle is composed of three parallel $2.5-\mathrm{cm}$ active-cooled needles spaced $0.5 \mathrm{~cm}$ apart and arranged in a triangular pattern. Needle cooling is ensured by peristaltic perfusion of chilled saline, which allows the electrode to maintain tip temperatures below $25^{\circ} \mathrm{C}$ during RF delivery. The generator was used in the auto-control mode generating a pulsed RF delivery for $15 \mathrm{~min}$ for each needle position. The auto-control mode allowed for the maximum power to be delivered until the impedance rose to $10 \Omega$ above the baseline value. When the current reached this level, it was automatically switched off for $15 \mathrm{~s}$; thereafter, it was switched on again generating pulsed RF known to increase size of lesions [9].

The Radiotherapeutics RF system used for these procedures included a Radiotherapeutics RF3000 generator with a maximal $200-\mathrm{W}$ power output that was linked to a $4-\mathrm{cm}$ Le Veen needle. Following deployment within the liver, we used the recommended algorithm that started with a RF power of $80 \mathrm{~W}$ that was further increased by $10 \mathrm{~W}$ per minute up to $130 \mathrm{~W}$. After $10 \mathrm{~min}$, if impedance had not increased, the RF power was again increased by $10-\mathrm{W}$ installments. The procedure was terminated when a marked increase in impedance, called "roll off," occurred.

The RITA RF system chosen for the experiment was the RITA RF model 1500 combined with 14-G Starburst XL needles. The needle was flushed before puncture by injecting saline through the lateral hub. After adequate needle positioning within the liver, the prongs were first deployed at $2-\mathrm{cm}$ length with the power set at $50 \mathrm{~W}$ until a temperature of $80^{\circ} \mathrm{C}$ was reached. Subsequently, the prongs were advanced forward until they were deployed at $3 \mathrm{~cm}$. At this length, a power of $70 \mathrm{~W}$ was used until a temperature of $105^{\circ} \mathrm{C}$ was reached. After further deployment of $4 \mathrm{~cm}$, the energy was set at $90 \mathrm{~W}$ until reaching $110^{\circ} \mathrm{C}$. Finally, after 5-cm deployment, RF energy was set at $110 \mathrm{~W}$ until a mean temperature of $110^{\circ} \mathrm{C}$ was reached and maintained for $7 \mathrm{~min}$. The generator was then turned off. If a temperature of $70^{\circ} \mathrm{C}$ was maintained for at least $30 \mathrm{~s}$ after switch off, the procedure was considered completed. If this temperature was not maintained, an additional 5-min RF delivery was performed with the temperature maintained at $110^{\circ}$. In addition, if the temperature of one prong remained low, the prongs were pulled back into pre-deployment position, rotated by $45^{\circ}$, then deployed again. Careful attention was paid during deployment to avoid pulling back of the needle.

\section{Statistical evaluation}

The volume of tissue destruction obtained by RF was evaluated by approximating the lesion to a sphere using the following formula: $\pi(\mathrm{DvxDtxDt}) / 6$. Because only one transverse diameter $\mathrm{Dt}$ to the 

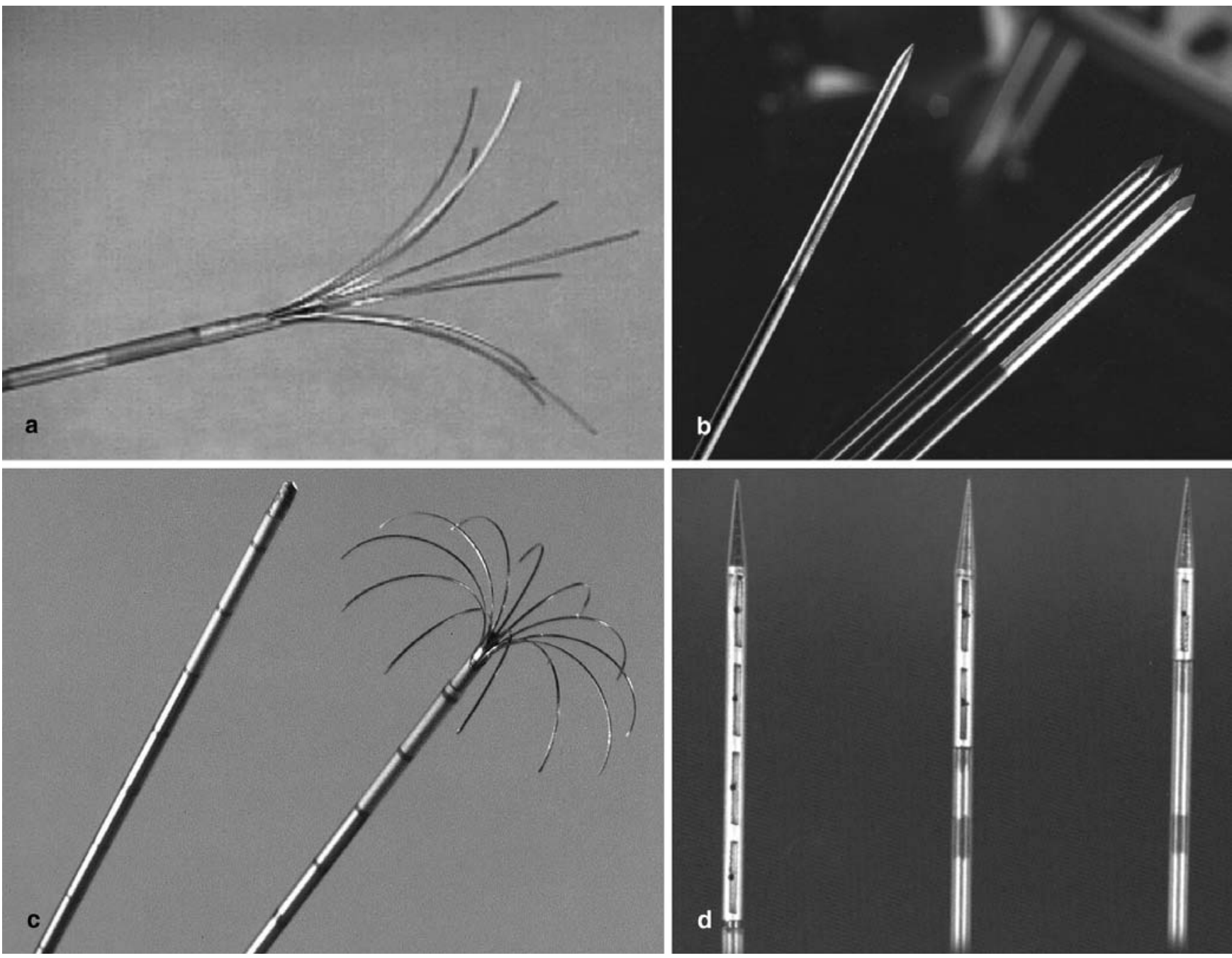

Fig. 1a-d Radio-frequency systems tested. a The tip of the RITA $14 \mathrm{G}$ Starburst XL needle tested with nine prongs deployed at the extremity. b The Radionics cooled-tip triple-cluster needle and single cooled-tip needle. c The Radiotherapeutics $4 \mathrm{~cm}$ Le Veen needle. d The Berchtold needle tips with side holes at the needle extremity

electrode could be measured, we used it twice in the formula assuming that the second transverse diameter measurement was similar to the first. Volume differences of in vivo lesions obtained with the multiple RF systems were compared using a one-way analysis of variance (ANOVA) test followed by an unpaired Student's $t$ test for group to group comparison. The same statistical evaluation was applied for ex vivo lesions. Lesion variability was compared between groups using crossed variance component analysis by $\mathrm{F}$ test of variance. Evaluation of the volume variation in each group was given by a variation coefficient calculated as follows: standard deviation/mean value.

Radio-frequency lesion shape was characterized by the ratio between the transverse and vertical diameter Dt/Dv. This ratio was compared between groups using one way ANOVA test, unpaired $t$ test was used for group-to-group comparison. Variability was compared between groups using crossed variance component analysis by $\mathrm{F}$ test of variance. Evaluation of the variation of volume in each group was given by a variation coefficient calculated as follows: standard deviation/mean value. Statistics were performed using the JMP statistical software (Statistical package 4.0, SAS Institute, Cary, N.C.)

\section{Results}

\section{In vivo RF lesions}

A total of 27 lesions could be completed in 21 pigs. Eight lesions each were created with the Radionics, Radiotherapeutics, and RITA systems. In three cases, when using the RITA device, the target temperature could not be reached in one of the thermocouples. This was thought to be due a large vessel in the vicinity of this array; therefore, the needle was re-deployed after a $45^{\circ}$ rotation of the device. No complications were observed using Radionics, RITA, and Radiotherapeutics system.

Regarding RF delivery time, RFA lesion creation took $15 \mathrm{~min}$ for Radionics and $19 \mathrm{~min}$ for Berchtold. The 

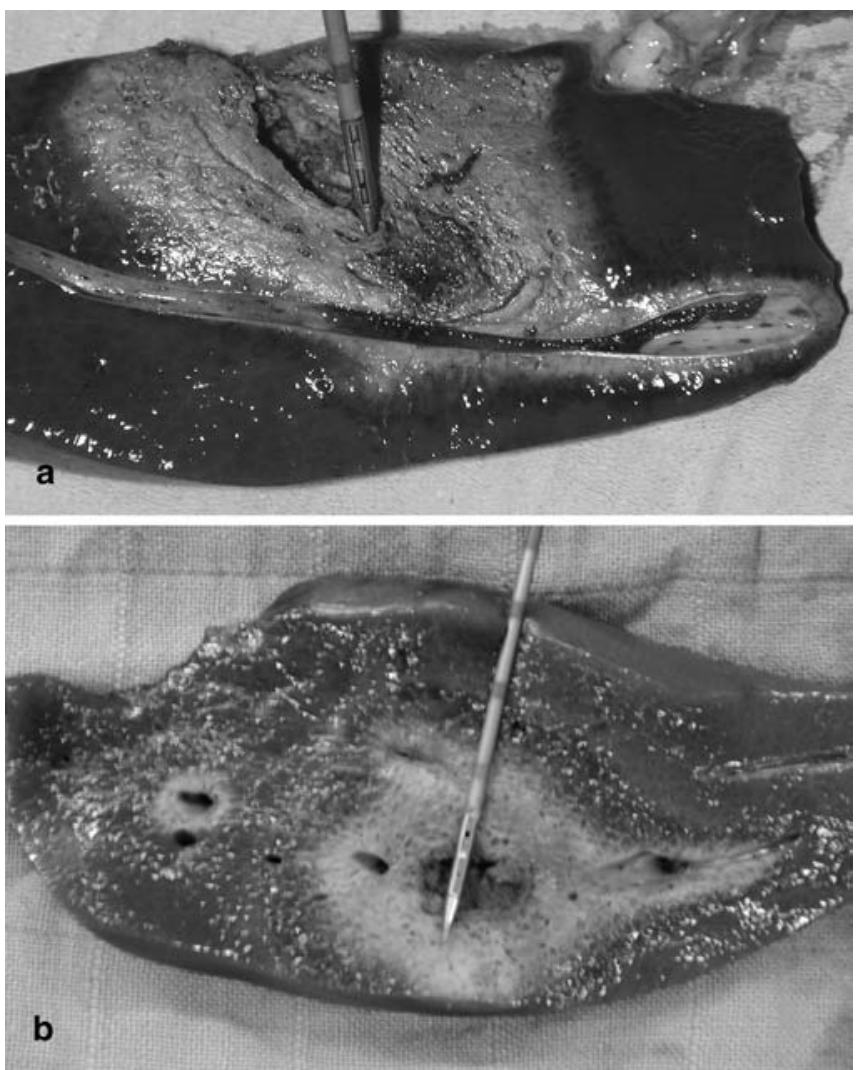

Fig. 2a, b Radio-frequency (RF) in vivo lesions created with the Berchtold system. a The RF lesion extending along the hepatic vein wall away from the RF lesion with fresh clots inside of the vein. b Another Berchtold in vivo lesion showing coagulation following vascular branches away from the RF lesion

mean RF delivery times for Radiotherapeutics was 17 min (standard deviation $2.2 \mathrm{~min}$, range 12-20 $\mathrm{min}$ ) and $25 \mathrm{~min}$ for RITA lesions (standard deviation $3.4 \mathrm{~min}$, range $15-32 \mathrm{~min}$ ).

With the Bertchtold system, only three lesions could be completed as 5 of 8 animals used with this unit died during the RF delivery. Analysis of RFA lesions in the 5 dead animals showed extension of the tissue coagulation along the vascular (portal or hepatic venous) pedicles (Fig. 2) with fresh clots in the hepatic veins in two cases. Because of this, RFA lesions created with this system did not allow for statistical analysis. Furthermore, one lesion created with the Berchtold system had extensive coagulation diffusing along the liver capsule (Fig. 3).

Lesions volumes obtained with RITA, Radiotherapeutics, and Radionics, were $39 \pm 4.3,42 \pm 10$, and $29 \pm 11 \mathrm{~cm}^{3}$, respectively (Table 1 ). Statistical differences were observed between these three groups using ANOVA test $(p<0.05)$. Group-by-group statistical comparison demonstrated statistical differences between Radiotherapeutics and Radionics $(p<0.05)$ and nearly significant difference between RITA and Radionics $(p=0.056)$. No statistical
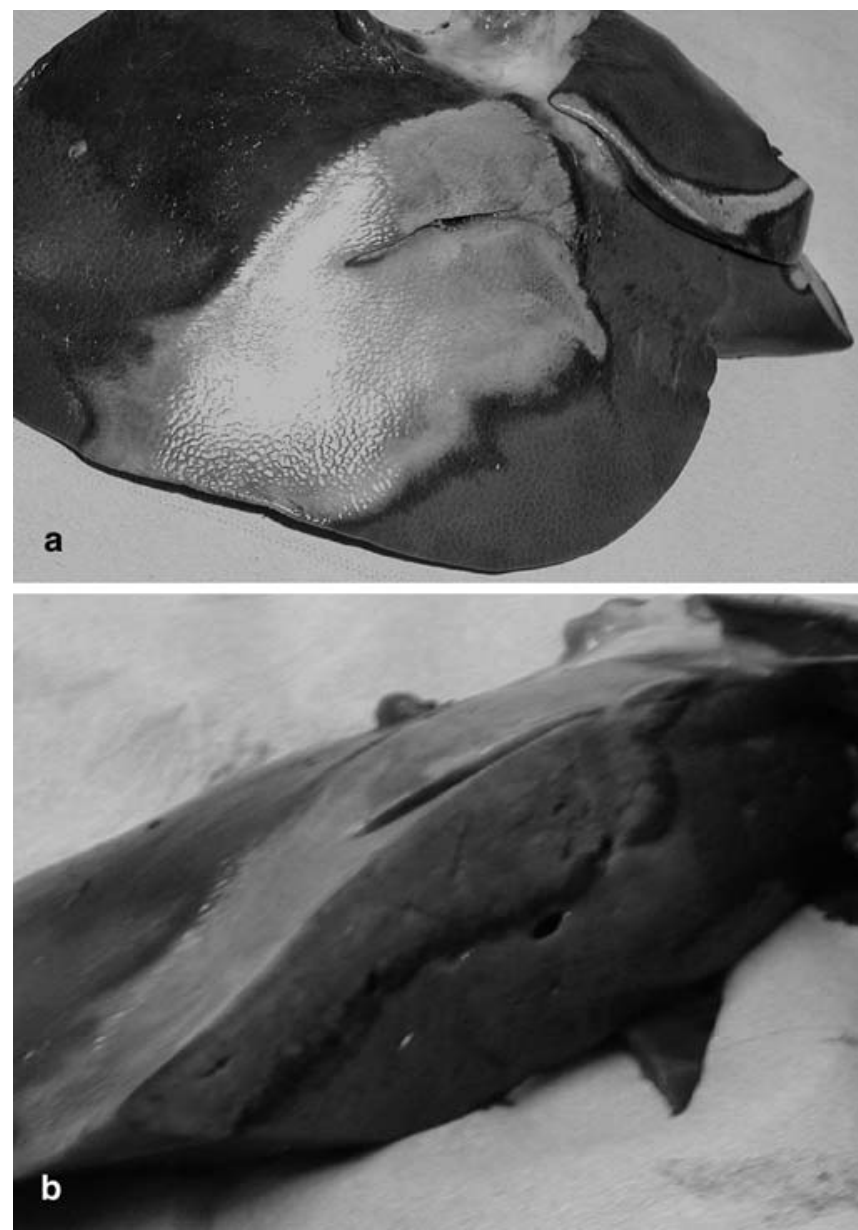

Fig. 3a, b The RF in vivo lesion created with the Berchtold system. a The anterior aspect of the liver shows a large lesions extended all along the liver surface. b The liver shows this ovoid lesion extending along the liver capsule

difference existed when RITA was compared with Radiotherapeutics using an unpaired $t$ test. F test analysis of variance found significant differences in variance between RITA, Radiotherapeutics, and Radionics $(p<0.05)$. Transverse diameter was statistically significantly different between RITA and Radiotherapeutics $(4.15 \pm 0.23$ and $4.3 \pm 0.5 \mathrm{~cm}$, respectively) and Radionics (3.62 \pm 0.41 ; $p<0.05)$.

The Dt/Dv ratio for Radiotherapeutics and RITA RF systems were $1.01 \pm 0.16$ and $0.97 \pm 0.1$, respectively, corresponding to near spherical shapes of the volume of coagulation (Fig. 4). More elongated lesions were demonstrated by Radionics and Bertchtold RF systems with $\mathrm{Dt} / \mathrm{Dvratios}$ of $0.88 \pm 0.09$ and $0.65 \pm 0.39$, respectively. There was not significant difference and there was not significant variance of Dv/Dt ratio using $\mathrm{F}$ test. 
Table 1 Mean volume of radio-frequency ablation (RFA) lesions created in pig liver in vivo

\begin{tabular}{|c|c|c|c|c|}
\hline & Bertchtod & Radionics & Radiotherapeutics & RITA \\
\hline Number & 3 & 8 & 8 & 8 \\
\hline Mean volume $\left(\mathrm{cm}^{3}\right) \pm \mathrm{SD}$ & $37 \pm 8$ & $29 \pm 11$ & $42 \pm 10$ & $39 \pm 4.3$ \\
\hline Volume variation coefficient (\%) & 22 & 37 & 24 & 11 \\
\hline Vertical diameter Dv $(\mathrm{cm})$ & $6.4 \pm 1.9$ & $4.16 \pm 0.6$ & $4.29 \pm 0.43$ & $4.3 \pm 0.29$ \\
\hline Transverse diameter W (cm) & $3.4 \pm 0.75$ & $3.62 \pm 0.41$ & $4.3 \pm 0.5$ & $4.15 \pm 0.23$ \\
\hline $\mathrm{Dt} / \mathrm{Dv}$ ratio $\pm \mathrm{SD}$ & $0.65 \pm 0.39$ & $0.88 \pm 0.09$ & $1.01 \pm 0.16$ & $0.97 \pm 0.1$ \\
\hline $\mathrm{Dt} / \mathrm{Dv}$ variation coefficient (\%) & 60 & 11 & 16 & 11 \\
\hline
\end{tabular}

Table 2 Mean volume and diameter ratio of ex vivo RFA lesions

\begin{tabular}{lcccc}
\hline & Bertchtold & Radionics & Radiotherapeutics & RITA \\
\hline Number & 8 & 8 & 8 & 8 \\
Mean volume $\left(\mathrm{cm}^{3}\right) \pm \mathrm{SD}$ & $67 \pm 24$ & $66 \pm 20$ & $55 \pm 13$ & $68 \pm 20$ \\
Volume variation coefficient $(\%)$ & 35 & 31 & 24 & 29 \\
Vertical diameter Dv $(\mathrm{cm})$ & $5.42 \pm 1.2$ & $5.56 \pm 0.72$ & $4.35 \pm 0.34$ & $5.94 \pm 0.62$ \\
Transverse diameter Dt $(\mathrm{cm})$ & $4.63 \pm 0.62$ & $4.69 \pm 0.44$ & $4.48 \pm 0.47$ & $4.61 \pm 0.45$ \\
Dt/Dv ratio \pm SD & $0.89 \pm 0.16$ & $0.84 \pm 0.06$ & $1.12 \pm 0.08$ & $0.78 \pm 0.03$ \\
Dt/Dv variation coefficient $(\%)$ & 18 & 7 & 6 & 4 \\
\hline
\end{tabular}

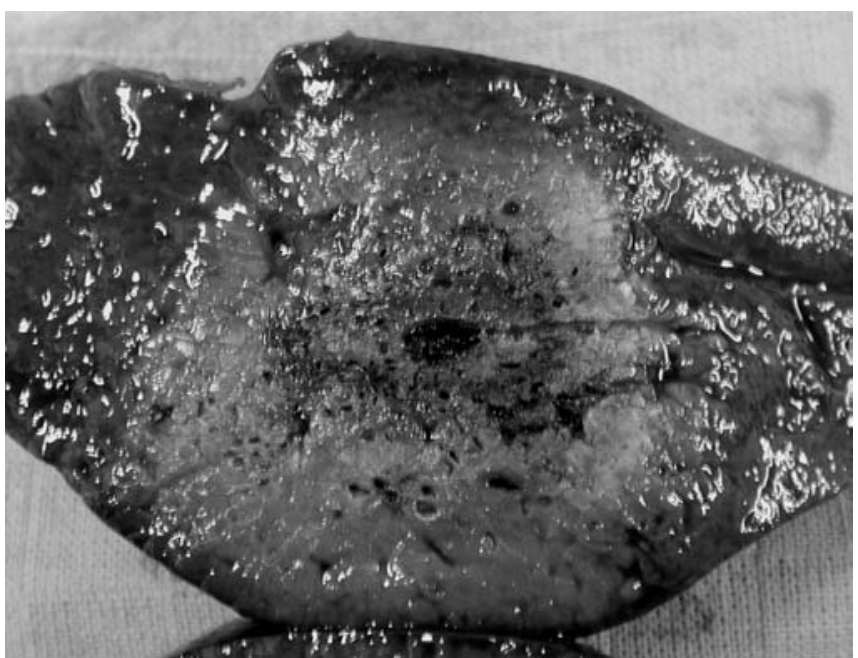

Fig. 4 The cut liver shows a 4.7-cm almost spherical in vivo lesion created with the RITA system

\section{Ex vivo RF lesions}

Thirty-two lesions could be performed in explanted calf liver (8 lesions for each system) Ex vivo lesion volumes ranged between 55 and $68 \mathrm{~cm}^{3}$ without statistical differences found between the four devices using the ANOVA test and Student's $t$ test (Table 2). Variation coefficients were also greater for lesion volume in ex vivo lesions as compared with in vivo lesions. Comparison of variance between groups of ex vivo lesion volume did not show any statistical significance, whereas comparison of variance of the Dv/Dt ratio in the same groups was highly significant $(p<0.00000001)$.

\section{Discussion}

Percutaneous RF tumor ablation has gained significant popularity during recent years. In particular, RFA has been advocated by many physicians as a primary treatment for selected patients with hepatocellular carcinoma. Recent reports have demonstrated that RFA is more efficacious than percutaneous ethanol injection (PEI) allowing for equivalent tumor control in fewer treatment sessions [14]; however, one limitation of this therapy is the size of RF lesions that can be obtained with one RF delivery. Although the efficacy of RFA is close to $100 \%$ for tumors less than $2 \mathrm{~cm}$ in diameter, the local recurrence rates are $\geq 20 \%$ for tumors larger than 2.5 or $3 \mathrm{~cm}$, and $\geq 50 \%$ for lesions larger than $5 \mathrm{~cm} \mathrm{[10].} \mathrm{In} \mathrm{the} \mathrm{same} \mathrm{way,}$ destruction of a lesion of $2 \mathrm{~cm}$ in size with a 1-cm safety margin corresponds to a RFA size in volume of $33.49 \mathrm{~cm}^{3}$. For a larger lesion of $3 \mathrm{~cm}$ in diameter with the same $1-\mathrm{cm}$ safety margin, this RFA volume should be $65.42 \mathrm{~cm}^{3}$. In our study the technological improvements allowed a theoretical destruction of lesions between 2 and $3 \mathrm{~cm}$ in diameter. One must consider that these improvements are important, but they are still far from what we may expect from a clinical point of view. New experimental developments recently demonstrated in experimental studies, such as combined wet cooled or wet expandable, may further improve RFA lesion size [9, $10,11]$.

Both RITA and Radiotherapeutics developed multiprobe needles that are able to deploy nine (Christmastree shaped) or ten (umbrella shaped) prongs, respectively. In our experimental study, these two systems provided the largest volume of tissue destruction. In addition, these systems created near-spherical lesions in vivo with 
(W/L) ratios of transverse to needle axis close to 1 . This finding is important for local tumor treatment as most hepatic tumors have a spherical shape. We did not observe any difference in terms of RF lesion volume between these two devices; however, repositioning of the needle was necessary in 3 cases with the RITA device, increasing the lesion creation time. The RITA RF system monitors intra-lesional temperature with five thermocouples. When a needle is positioned close to a vessel, the temperature does not increase in this area. As a consequence, the needle position can be modified to avoid this limitation. This may explain, in part, the reproducible volumes obtained with this device as compared with the Radiotherapeutics system which monitors treatment with an impedance control mechanism.

Another strategy that can be used to increase RF lesion size is to minimize carbonization ("charring") of tissue around needle tip. This can be done through a continuous perfusion of cold saline within the needle lumen as proposed by Radionics; however, our study shows that in vivo lesions produced by Radionics were slightly smaller and more ovoid in shape than the lesions produced by RITA or Radiotherapeutics devices.

Bertchtold applies another concept to increase lesion size with RF. Isotonic saline can be infused into the tissue through holes at the extremity of the needle, thereby increasing electrical conductivity in the needle vincinity [10]. The protocol used in our investigation and recommended by the Berchtold company killed 5 of 8 animals within the first $3 \mathrm{~min}$ of RF delivery with perfusion. At present, we have no definite explanation for these deaths; however, livers removed from 4 of the 8 pigs treated with this device show coagulation running along the large hepatic veins and away from the RF lesion. This finding suggested diffusion of the heated saline along the outside wall of these veins. Moreover, in 2 cases, clots were found in the hepatic veins. For this reason, we considered pulmonary embolism as a possible explanation for their deaths; however, in clinical series, such complications have never been reported, to our knowledge in the literature [11]; however, the shape of the lesions were irregular as previously observed [12, 13].

Our study supports the recent finding of Goldberg et al. who evaluated the consequences of hyperosmolar saline injection [14], mentioning that shape of lesions were irregular and hardly predictable, especially when large amounts of saline ( $>25 \mathrm{ml}$ ) were injected. In our study, a total amount of $25 \mathrm{ml}$ was used as recommended by the manufacturer. This large amount of saline may explain for spread of coagulation distally to the RF lesions along the vascular bed or under the capsule.

This study also demonstrates that using ex vivo comparisons of RF systems brings no significant valuable information to the in vivo efficacy of these systems. Indeed, despite statistical differences that were observed in vivo, no differences were observed ex vivo. Moreover, the variation coefficients of RF lesion volumes were higher for ex vivo than for in vivo lesions for RITA and Radiotherapeutics systems.

This study does have some limitations. Firstly, it is not known whether the results of this animal liver model accurately predict the results of treating liver tumors in patients [15]. Differences in tissue vascularization, impedance, and heterogeneity in tumors may modify the results that we observed. Secondly, we have no definite explanation for the deaths of 5 animals using Bertchtold device. We hypothesized that the deaths were due to massive pulmonary emboli on the basis of hepatic vascular thrombosis. Since that hypothesis was retrospectively supposed on the basis of liver pathological studies, we did not take the opportunity to check the lung at autopsy. This complication has never been described in human studies with this unit; however, literature regarding use of the device is limited. Thirdly, we did not use the most recent expandable needles developed by RITA and Radiotherapeutics (Starburst Xli and Le Veen 6.0, respectively) since they were not yet available. Fourth is the size of pig liver. Even when using the largest pigs available in our institute, the thickness of the livers rarely exceeded $6 \mathrm{~cm}$ due to their leafy anatomy. For this reason, it was possible to perform only one to three RF lesions per animal resulting in the relatively low number of lesions in our study. Fifthly, systems were run according to each manufacturer's specifications. Their recommendations may or may not have represented the greatest degree of optimization possible. Superiority of one system over another only reflects the situation in the experimental conditions described at the moment of the study. This may rapidly change in the future due to new needle or protocol improvements.

\section{Conclusion}

In this experimental study, we did not observe differences between expandable needles systems in vivo. Cooled needles produced slightly smaller lesions with a more ovoid shape. Improvements in needles and delivery protocols are still mandatory to treat with safety margins tumors larger than $3 \mathrm{~cm}$ in diameter.

Acknowledgement We thank P. Frascarolo for his precious help in the statistical evaluation of our results. 


\section{References}

1. Goldberg SN, Dupuy DE (2001) Image-guided radiofrequency tumor ablation: challenges and opportunities, part I. J Vasc Interv Radiol 12:1021-1032

2. McGahan JP, Griffey SM, Budenz RW, Brock JM (1995) Percutaneous ultrasound-guided radiofrequency electrocautery ablation of prostate tissue in dogs. Acad Radiol 2:61-65

3. de Baere T, Denys A, Johns-Wood B et al. (2001) Radiofrequency liver ablation: experimental comparative study of water-cooled vs expandable systems. Am J Roentgenol 176:187-192

4. Goldberg SN, Gazelle GS, Solbiati L, Rittman WJ, Mueller PR (1996) Radiofrequency tissue ablation: increased lesion diameter with a perfusion electrode. Acad Radiol 3:636-644

5. Goldberg SN, Hahn PF, Tanabe KK et al. (1998) Percutaneous radiofrequency tissue ablation: Does perfusion-mediated tissue cooling limit coagulation necrosis? J Vasc Interv Radiol 9:101-111
6. Goldberg SN, Solbiati L, Hahn PF et al. (1998) Large-volume tissue ablation with radiofrequency by using a clustered, internally-cooled electrode technique: laboratory and clinical experience in liver metastases. Radiology 209:371-379

7. Curley SA, Izzo F, Delrio P et al. (1999) Radiofrequency ablation of unresectable primary and metastatic hepatic malignancies: results in 123 patients (see comments). Ann Surg 230:1-8

8. Curley SA, Izzo F, Ellis LM, NicolasVauthey J, Vallone P (2000) Radiofrequency ablation of hepatocellular cancer in 110 patients with cirrhosis. Ann Surg 232:381-391

9. Goldberg SN, Stein MC, Gazelle GS, Sheiman RG, Kruskal JB, Clouse ME (1999) Percutaneous radiofrequency tissue ablation: optimization of pulsed radiofrequency technique to increase coagulation necrosis. J Vasc Interv Radiol 10:907-916

10. Livraghi T, Goldberg SN, Lazzaroni S et al. (2000) Hepatocellular carcinoma: radiofrequency ablation of medium and large lesions. Radiology 214:761-768

11. Gangi A, Dupas B, Guth S et al. (2001) Application des électrodes humides de radiofréquence dans le traitement des tumeurs hépatiques. J Radiol 82:1430
12. Livraghi T, Goldberg SN, Monti F et al. (1997) Saline-enhanced radiofrequency tissue ablation in the treatment of liver metastases. Radiology 202:205-210

13. Miao Y, Ni Y, Mulier S et al. (1997) Ex-vivo experiment on radiofrequency liver ablation with saline infusion through a screw-tip cannulated electrode. J Surg Res 71:19-24

14. Goldberg SN, Ahmed M, Gazelle GS et al. (2001) Radio-frequency thermal ablation with $\mathrm{NaCl}$ solution injection: effect of electrical conductivity on tissue heating and coagulation-phantom and porcine liver study. Radiology 219:157-165

15. Denys AL, de Baere T, Mahe $\mathrm{C}$ et al. (2001) Radiofrequency tissue ablation of the liver: effects of vascular occlusion on lesion diameter and biliary and portal damages in a pig model. Eur Radiol 11:2102-2108

16. Goldberg S, Hahn P, Halpern E, Fogle R, Gazelle G (1998) Radiofrequency tissue ablation: effect of pharmacologic modulation of blood flow on coagulation diameter. Radiology 209:761-767 